\title{
Anabases
}

ANABASES Traditions et réceptions de l'Antiquité

$27 \mid 2018$

Varia

\section{Restituer l'espace domestique à Herculanum grâce aux outils informatiques de reconstruction virtuelle : enjeux et problèmes}

Alexandra Dardenay

\section{OpenEdition}

Journals

Édition électronique

URL : http://journals.openedition.org/anabases/6668

DOI : $10.4000 /$ anabases.6668

ISSN : 2256-9421

Éditeur

E.R.A.S.M.E.

\section{Édition imprimée}

Date de publication : 1 avril 2018

Pagination : 41-51

ISSN : 1774-4296

\section{Référence électronique}

Alexandra Dardenay, «Restituer l'espace domestique à Herculanum grâce aux outils informatiques de reconstruction virtuelle : enjeux et problèmes », Anabases [En ligne], 27 | 2018, mis en ligne le 01 avril 2020, consulté le 20 janvier 2021. URL : http://journals.openedition.org/anabases/6668 ; DOI : https:// doi.org/10.4000/anabases.6668 


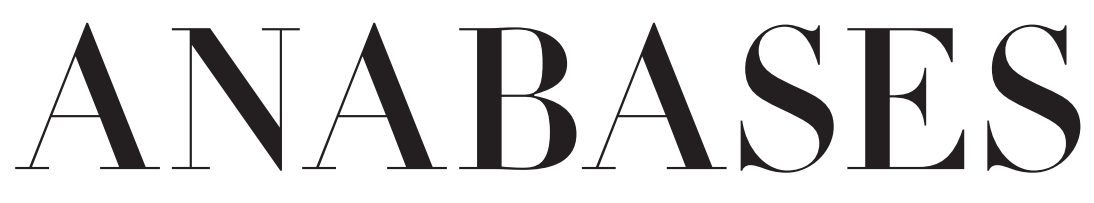

Traditions et Réceptions de l'Antiquité

$$
\begin{aligned}
& \text { No27 } \\
& 2018
\end{aligned}
$$

E.R.A.S.M.E.

Université Toulouse - Jean Jaurès 



\section{Sommaire}

$\mathrm{N}^{\circ} 27-2018$

\section{Historiographie et identités culturelles}

\section{Sébastien Cazalas}

“ et pour ce recite Valere... ». La place de l'Antiquité dans l'arsenal

intellectuel d'un grand prélat français du XVe siècle :

Jean Juvénal des Ursins (I388-I473) . . . . . . . . . . . . . . . . . . . II

Loic Marcou

La réception de l'Antiquité dans l'œuvre de la femme de lettres grecque

Rhéa Galanaki. . . . . . . . . . . . . . . . . . . . . .

\section{Traditions du patrimoine antique}

« Restituer Herculanum II. Des archives de fouilles aux restitutions 3D

Alexandra DARDENAY

Introduction : restituer l'espace domestique à Herculanum

grâce aux outils informatiques de reconstruction virtuelle ;

enjeux et problèmes. . . . . . . . . . . . . . . 4

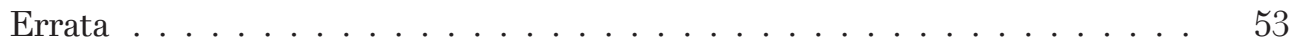

Carla Marotta

L’utilisation des données archivistiques dans l'enquête archéologique : nouvelles découvertes sur la maison d'Argos . . . . . . . . . . . . . . .

Emmanuelle Rosso

Restituer l'emplacement des statues dans les édifices d'Herculanum?

Problèmes de méthode . . . . . . . . . . . . . . . 7I 
Hélène Eristov, Marie-Laure Maraval

Restituer les décors perdus de la maison de Neptune et d'Amphitrite :

enquête, méthodes, résultats . . . . . . . . . . . . 9 9I

Maud Mulliez

Restauration numérique des peintures murales de la maison

de Neptune et Amphitrite à Herculanum : de l'expérimentation

matérielle à l'intégration des données dans un modèle 3D . . . . . . . Io3

\section{Archéologie des savoirs}

Nicolas SIRON

L'histoire de Philippidès d'Hérodote à Lucien.

Une incursion dans l'atelier du mythe . . . . . . . . . . . . . . . . Iog

Constantin Raios

Le coracin (xo@axîvos) du lac de Tibériade (F. Josèphe, B.J. III, 520) . . .

\section{Actualités et débats}

Claude Azıza

Antiquités parallèles (8). Le syndrome du sein droit . . . . . . . . . . . . I6I

Elisabeth DÉcultot

Winckelmann. Moderne Antike / Winckelmann. Modern Antiquity.

Exposition, Weimar, Neues Museum, 7 avril-2 juillet 20I7 . . . . . . . I67

\section{Lire, relire la bibliothèque des sciences de l'Antiquité}

Cyrielle LANDrea

Jérôme Carcopino et la mémoire perdue

de M. Valerius Messalla Corvinus (cos. 3г av. J.-C.) . . . . . . . . . . . . $\quad$ I77

Jérôme CARCopino

Notes biographiques sur M. Valerius Messala Corvinus . . . . . . . . . I I85

\section{L'atelier de l'histoire : chantiers historiographiques}

L'Atelier des doctorants (coordonné par Adeline Grand-Clément) (15)

Jonathan Pérez Mostazo

Cantabri aut vascones. La reception de la Antigüedad

en la cultura histórica vasca del siglo XIX . . . . . . . . . . . . . . . . . 209 


\section{Comptes rendus}

William H.F. Altman (éd.)

Brill's Companion to the Reception of Cicero (A. A. Raschieri). . . . . . . . 2 2I9

Anthony A. Barrett, Elaine Fantham, John C. Yardley

The Emperor Nero. A Guide to the Ancient Sources (É. Deniaux) . . . . . . 22I

Pascale Barthélémy et Violaine sébillotte cuchet (éd.)

Clio. Femmes, Genre, Histoire $\mathrm{n}^{\circ} 43$ : Citoyennetés (M. L. Napolitano) . . . 222

Frédéric Colin, Olivier Huck, Sylvie Vanseveren (éd.)

Interpretatio. Traduire l'altérité culturelle dans les civilisations

de l'Antiquité (Cl. Joncheray) . . . . . . . . . . . . . . . . . . . . 224

Angus Fletcher

Comic Democracies. From Ancient Athens

to the American Republic (A. de Crémoux). . . . . . . . . . . . . . . . . . 226

David Hernández de la Fuente

El despertar del alma. Dioniso y Ariadna: mito y misterio (A. Iriarte) . . . . 228

Ted Kaizer (éd.)

Religion, Society and Culture at Dura-Europos (P. G. Michelotto) . . . . . 229

Jacques Le Goff, Jean-Pierre Vernant

Dialogue sur l'histoire. Entretiens avec Emmanuel Laurentin (C. Lucci). . 23I

Justine Mac Connell, Edith Hall

Ancient Greek Myth in World Fiction since ig8g (A. de Crémoux) . . . . . . 233

Angelo Mazzocco, Marc Laureys (éd.)

ANew Sense of the Past. The Scholarship

of Biondo Flavio (I3g2-I463) (C. Lucci). . . . . . . . . . . . . . . . . . . . . . 234

Seth L. Schein

Homeric Epic and Its Reception. Interpretative Essays (S. Sistac) . . . . . . 236

Arbogast SснмiтT

Wie aufgeklärt ist die Vernunft der Aufklärung?

Eine Kritik aus aristotelischer Sicht (L. Calvié) . . . . . . . . . . . . . . . . 238

Richard Sorabu (éd.)

Aristotle Re-Interpreted. New Findings on Seven Hundred Years

of the Ancient Commentators (P. Butti de Lima) . . . . . . . . . . . . . . . 239

Laura SwiFT

Greek Tragedy. Themes and Contexts (G. Aujac) . . . . . . . . . . . . . . 24

Zara Martirosova TorLone

Vergil in Russia. National Identity and Classical Reception

(D. Millet-Gérard) . . . . . . . . . . . . . . . . . . 243 
Jürgen von UNGERN-STERNBERG

Les chers ennemis. Deutsche und französische

Altertumswissenschaftler in Rivalität und Zusammenarbeit (L. Calvié) . . 2444

Robert W. WALLACE

Reconstructing Damon: Music, Wisdom Teaching, and Politics

in Perikles'Athens (A. Brancacci) . . . . . . . . . . . . . . . . . . . . . 246

T. P. Wiseman

The Roman Audience : Classical Literature as Social History (C. Landrea) 249

Résumés . . . . . . . . . . . . . . . . . . . . . . . . . . 253

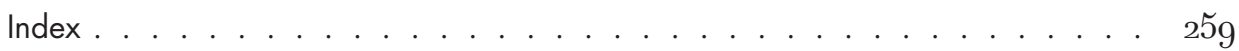




\section{Traditions}

\section{du patrimoine antique}

Restituer Herculanum II.

Des archives de fouilles aux restitutions 3D 



\section{Restituer l'espace domestique à Herculanum grâce aux outils informatiques de reconstruction virtuelle : enjeux et problèmes}

Alexandra DARDENAY

I es pensionnaires de l'Académie de France à Rome, lauréats du Grand Prix de Rome, se voyaient, pour certains, commander une étude complète d'un monument de Pompéi. Leur envoi devait contenir un plan général, des coupes, des dessins de détails architecturaux, ainsi que des propositions de

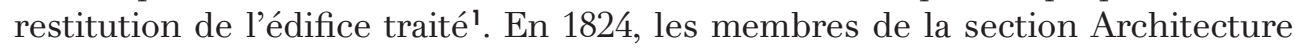
des Beaux-Arts définissent précisément l'exercice de la restitution, qui doit être “ la conjecture la plus probable appuyée d'autorités, de la forme, de la figure et des proportions d'un monument, aujourd'hui en ruines, et de ce qu'il pouvait être au temps de sa splendeur ${ }^{2}$. Les propositions de reconstruction, que l'on souhaite toutefois étayées par des données architecturales et matérielles fondées, sont donc laissées à l'appréciation des pensionnaires missionnés. La restitution de la polychromie et du décor pictural sont le plus souvent mises en œuvre par les architectes, en dépit des nombreuses lacunes provoquées par l'usure du temps ou les prélèvements. Pour le traitement de cette partie du travail, une part plus

1 Pompéi, travaux et envois des architectes français au XIXe siècle, catalogue de l'exposition, Paris, 1981 ; E. Brugerolles et H. Vuillefroy de Silly, s.v. “ FélixEmmanuel Callet », in M. Osanna, M. T. Caracciolo et L. Gallo (dir.), Pompéi et l'Europe (1748-1943), Milan, 2015, p. 190-191.

2 Rapport de l'Institut sur les ouvrages envoyés par les Architectes pensionnaires de l'Académie de France à Rome, Rome, Archives de la Villa Médicis, carton n 30, cité dans Pompéi, travaux et envois, 1981, p. 75. 
importante est généralement accordée à l'imagination et il n'est pas rare que certaines libertés soient prises par rapport aux vestiges. Dans les études réalisées par Jean-Baptiste Cicéron Le Sueur (1794-1883) - lauréat du Grand Prix de Rome en 1819 - lors de son séjour en Italie, 156 œuvres sont consacrées à Pompéi. Le macellum (que l'on prenait alors pour un Panthéon) a plus particulièrement retenu son attention, et l'artiste s'est attaché à la restitution du décor. En dépit du bon état de conservation de ces peintures - relevant stylistiquement du IV ${ }^{\mathrm{e}}$ style scénographique - il n'en livre toutefois pas une étude fidèle. L'articulation générale de la composition est respectée, mais Le Sueur modifie substantiellement les proportions, inverse les couleurs et transforme le schéma iconographique du tableau central figurant le mythe de Io et Argos, ajoutant, par exemple, un glaive dans la main d'Argos. D'un pensionnaire à l'autre, ou même d'une étude à l'autre, on oscille ainsi entre relevés rigoureux et fantaisies architecturales ou décoratives ${ }^{3}$.

Aujourd'hui encore, à l'heure où les dessins informatiques remplacent peu à peu relevés graphiques et aquarelles, archéologues et architectes investis dans des restitutions de monuments antiques sont confrontés à une succession de choix et arbitrages, afin de respecter au plus près l'intégrité d'un édifice et de son décor, tout en palliant les lacunes. Les questions et problèmes auxquels étaient soumis les pensionnaires de l'Académie de France à Rome, au xix ${ }^{e}$ siècle, restent donc entières, même si les réponses apportées sont aujourd'hui différentes : comment restituer, et surtout, jusqu'où restituer?

L'équipe du programme VESUVIA, qui souhaite proposer aux chercheurs et au grand public un modèle informatique 3D de l'habitat herculanéen, a dû affronter de tels questionnements. L'examen des archives liées aux fouilles du site, depuis l'époque moderne, a constitué une première étape importante du travail et a permis d'élaborer des restitutions graphiques du décor des édifices ${ }^{4}$. La deuxième phase de l'enquête a pris un virage plus anthropologique et social, avec la tentative de “ réaménagement » des espaces habités, à l'aide d'élément de mobilier et de l'instrumentum en général. Cette partie du travail de restitution apparaît comme plus délicate et aléatoire que la restitution architecturale, dans la mesure où les vestiges fragiles et périssables qui composent l'ameublement et l'équipement d'une maison ne sont pas toujours découverts in situ.

3 E. Brugerolles et H. Vuillefroy de Silly, s.v. “ Jean-Baptiste Cicéron Le Sueur », in Pompéi et l'Europe, p. 198-199.

4 Cet aspect du travail de restitution a été envisagé dans le précédent dossier Restituer Herculanum, paru dans Anabases 26 (2017). 


\section{Analyse de la sphère domestique dans la perspective d'une restitution 3D}

À Herculanum, l'un des premiers à entreprendre cette enquête fut Stephan Mols, à l'occasion d'une thèse doctorale portant sur le mobilier en bois mis au jour sur le site ${ }^{5}$. Son initiative fut complétée par celle de Nicolas Monteix, dont la thèse sur Les lieux de métier à Herculanum fut l'occasion de recontextualiser l'instrumentum mis au jour au $\mathrm{XX}^{\mathrm{e}}$ siècle dans les boutiques et ateliers ${ }^{6}$. Dans le présent dossier, Emmanuelle Rosso révèle les difficultés de mise en œuvre d'une telle tentative, quand elle s'applique aux sculptures et reliefs découverts à Herculanum depuis l'époque des Bourbons, à une époque, donc, où la localisation des découvertes était rarement enregistrée. Carla Marotta, dont les recherches préliminaires sur la casa d'Argo sont également proposées dans ce volume, met en évidence toutes les entraves rencontrées au cours de l'enquête pour retrouver les objets mis au jour dans cette vaste demeure. En effet, les prélèvements réalisés aux XVIII ${ }^{\mathrm{e}}$ et XIX ${ }^{\mathrm{e}}$ siècles ont rejoint les collections royales, puis celles du musée de Naples, sans que soient enregistrées des informations relatives à leur provenance. Or, si les peintures et mosaïques peuvent être parfois recontextualisées grâce aux indices architecturaux, aux traces liées à leur dépose, aux similitudes stylistiques et iconographiques avec les fragments de décor laissés in situ, le prélèvement des sculptures, du mobilier et de l'instrumentum n'a, quant à lui, laissé aucune trace permettant de le rattacher à son environnement originel. La situation est moins désespérée s'agissant des édifices fouillés au XX ${ }^{\mathrm{e}}$ siècle, sous la tutelle d'A. Maiuri, dans la mesure où les journaux de fouille enregistrent, en principe, les découvertes de mobilier? Et parfois, quoique rarement, des photographies réalisées durant la phase de dégagement des structures documentent l'emplacement de quelques meubles.

Dans la perspective d'une remise en contexte du mobilier pour une restitution 3D des maisons d'Herculanum, les deux principaux types de problèmes à affronter sont les suivants. En premier lieu, on doit s'interroger sur l'apparence initiale des meubles découverts carbonisés ${ }^{8}$. De quels matériaux étaient-ils

5 S.T.A.M. Mols, Wooden furniture in Herculaneum: form, technique and function, Amsterdam, 1999.

6 N. Monteix, Les lieux de métier : boutiques et ateliers d'Herculanum, Rome, 2010.

7 Même si les travaux de Nicolas Monteix montrent que ces enregistrements n'ont pas toujours la fiabilité souhaitée : voir N. MonteIx, “Les archives des fouilles d'Herculanum au XX ${ }^{\mathrm{e}}$ siècle: analyse critique et pistes d'exploitation ", Anabases 26 (2017), p. 143-154.

8 Dans sa thèse citée plus haut, Stephan Mols avait ainsi réfléchi à la reconstruction graphique du mobilier mis au jour à Herculanum (Wooden furniture in Herculaneum, 
composés, outre le bois (ivoire, pierre, métal...)? Quelle était la couleur initiale des essences de bois utilisées, et des matériaux éventuellement utilisés comme éléments rapportés ? Les meubles étaient-ils peints et si oui, sur quelle(s) partie(s), et avec quelle(s) couleur(s) ? En second lieu, on doit tenter de déterminer leur emplacement exact, lorsque la maison était habitée et/ou au moment de l'éruption. De plus, les journaux de fouille ne comportent pas toujours d'information relative à la localisation précise. Certains meubles furent même repositionnés sur le site, sans souci d'exactitude, pour mettre en valeur les demeures nouvellement fouillées, selon des “ mises en scène » orchestrées par A. Maiuri ${ }^{9}$.

Pour résoudre la question de la morphologie des meubles, le recours à la comparaison est d'un grand secours, essentiellement avec le mobilier représenté dans l'iconographie antique, puisque les pièces de mobilier de bois ne nous parviennent généralement pas parfaitement intactes ${ }^{10}$. Des comparaisons utiles, bien qu'un peu plus lointaines, peuvent aussi être proposées avec les copies en pierre sculptées et peintes de mobilier grec mises au jour dans des demeures ou des tombes hellénistiques" ${ }^{11}$. Mais la part laissée à l'imagination demeure très importante pour ce qui tient à l'apparence exacte des tissus et coussins polychromes qui recouvraient les banquettes.

La question des portes est également cruciale, vue leur importance dans l'articulation de l'espace domestique. Mais comment les restituer, alors que presque toutes ont brûlé ? Il existe des moulages de portes réalisés à Pompéi, par Fiorelli puis par ses disciples ${ }^{12}$. Les formes sont donc connues, et on sait qu'elles étaient en bois, parfois armées de métal. On ignore en revanche quel traitement de surface final elles avaient reçu. Tout laisse supposer qu'elles étaient peintes, car les Romains ne laissaient, a priori, pas souvent les matériaux bruts, ni dans

1999). Plus récemment, l'ouvrage de J.-P. Adam, La maison romaine, Paris, 2012, propose des reconstructions infographiques réussies.

9 Sur ces mises en scène et les déplacements de mobilier nécessaires pour les opérer : Monteix, Les lieux de métier (supra n. 6), p. 20-21, notamment fig. 3 (la « chambre de la brodeuse »).

10 D. Andrianou, "Chairs, Beds, and Tables: Evidence for Furnished Interiors in Hellenistic Greece ", Hesperia: The Journal of the American School of Classical Studies at Athens 75.2 (2006), p. 219-266.

1 D. Andrianou, The furniture and furnishings of ancient Greek houses and tombs, Cambridge, 2009 ; Andrianou, Chairs, Beds, and Tables (supra n. 10) ; A.-M. GuimierSorbets, "Mobilier et décor des tombes macédoniennes ", in Recherches récentes sur le monde hellénistique: actes du colloque international organisé à l'occasion du 60 anniversaire de Pierre Ducrey, Bern, 2001, p. 217-229.

12 G. Fiorelli, Gli scavi di Pompei dal 1861 al 1872, Napoli, 1873. 
l'architecture, ni dans le décor domestique. Stephan Mols suggère que les portes d'Herculanum étaient généralement peintes en rouge ocre et que parfois elles se fondaient complètement dans le décor de la paroi ${ }^{13}$.

\section{Mobilier, instrumentum et fonction des espaces dans la maison romaine}

Dans un article publié en $1993^{14}$, P. Allison présente les premiers résultats de ses travaux sur le rôle de l'étude des artefacts dans l'interprétation des espaces de la maison romaine, à partir de l'exemple de Pompéi. L'auteur insiste sur le poids des sources littéraires romaines sur nos usages en matière de dénomination des pièces. Elle souligne que trois principaux auteurs - Varron, Vitruve et Pline le jeune - sont pour l'essentiel responsables de notre compréhension moderne de la nomenclature et de l'interprétation de la fonction des pièces de la maison romaine. P. Allison invite alors à adopter une autre approche, qui permette de lier culture matérielle et fonctionnalité des espaces. Selon elle, c'est l'instrumentum, plus que la planimétrie, qui permet de comprendre la fonction d'une pièce. Elle tente alors de relocaliser, autant que possible, dans chaque espace de Pompéi, le mobilier et tous les artefacts qui s'y trouvaient au moment de l'éruption. Pour cela elle a entrepris une analyse des archives de fouilles de Pompéi, afin de retrouver l'emplacement de découverte du mobilier dans plusieurs habitations, sélectionnées selon un échantillonnage. Ses travaux ont donné naissance à une nouvelle grille de lecture de l'espace domestique pompéien, expurgée du vocabulaire latin, qui, bien que pertinente, se révèle finalement très conceptuelle. Cette typologie des pièces, qui identifie vingt-deux types d'espaces, pose un problème à l'usage, car il est difficile de se l'approprier et de la mémoriser ${ }^{15}$. L'hypercriticisme vis-à-vis du vocabulaire vitruvien - dont $\mathrm{P}$. Allison se fait l'écho - a généré un malaise des chercheurs dans la désignation des espaces de la maison romaine. La phase historiographique que

13 Dans les restitutions graphiques ou infographiques, on note que les portes sont souvent représentées de la couleur du bois brut (AdAM, La maison romaine, p. 7479), voire escamotées (B.A. Bergmann, Roman frescoes from Boscoreale: the Villa of Publius Fannius Synistor in reality and virtual reality, New York, 2010, p. 25-26). La découverte, à Pompéi, d'un fragment de porte peinte en prolongement du décor de la paroi, confirme l'existence de ce type de trompe l'œil (N. MonteIx et al., " Pompéi, Pistrina ", Chron. Act. Archéologiques L'École Fr. Rome [2013], fig. 7 [consulté le 6 octobre 2017, URL $=$ https://cefr.revues.org/954]).

14 P.M. Allison, "How do we identify the use of space in Roman housing? ", in E.M. Moormann (dir.), Functionnal and Spatial Analysis of Wall Painting. Proceedings of the Fifth International Congress on Ancient Wall Painting. Amsterdam, September 1992, 1993, p.1-8 ; repris et développé dans P.M. Allison, Pompeian households: an analysis of material culture, Los Angeles, 2004, chap.7, p. 161-177.

15 P.M. Allison, Pompeian households (supra n. 14), p. 64, tableau 5a. 
nous vivons actuellement est caractérisée par une période de flottement, entre la déconstruction de la grille de lecture “ canonique », d'origine vitruvienne, et la quête d'une solution onomastique pérenne pour désigner les pièces et espaces constituant les habitations. Aucune solution pleinement satisfaisante n'a, pour l'heure, émergée.

La relation entre culture matérielle et fonction des espaces est, bien entendu, au centre de l'enquête, dans la mesure où les objets sont supposés nous donner des indications sur les activités humaines qui pouvaient se dérouler dans une pièce. À cet égard, la démarche vient, toutefois, se confronter à un malentendu que l'on pourrait qualifier d'historique sur l'état de la cité d'Herculanum au moment de l'enfouissement, puisque l'on considère généralement que l'intégralité du mobilier et de l'instrumentum des maisons a été abandonnée sur place ${ }^{16}$. En effet, toute interprétation de la fonction des pièces reposant sur le mobilier mis au jour sur le site par les fouilleurs de Maiuri est fondée sur l'idée présupposée que les habitants d'Herculanum ont été surpris par l'éruption en ce matin d'octobre $79^{17}$ et ont abandonné leurs habitations précipitamment, laissant tous leurs biens derrière eux. Or, les choses ne se sont sans doute pas déroulées ainsi ; au contraire, de nombreux habitants ont, probablement, préparé et anticipé leur départ ${ }^{18}$.

16 P. Allison a été la première à refuser l'idée que Pompéi soit une simple photographie (P.M. Allison, "Artefact Assemblages: Not the Pompeii Premise », in E. Herring et al. (dir.), Papers of the fourth Conference of Italian archaeology, London, 1992, p. 49-56. Elle alerte sur ce problème épistémologique et envisage longuement les scénarios possibles dans deux chapitres intitulés " Considering Pompeian House Content (Allison, Pompeian households, p. 3-8) et " Nature of evidences » (Allison, Pompeian households, p. 10-26). Elle a proposé toutefois une méthodologie ingénieuse pour différencier les maisons qui étaient effectivement habitées à Pompéi en 79 de celles qui étaient vides ou abandonnées. Sa méthode repose surtout sur l'inventaire de l'instrumentum lié à la préparation des repas (car elle souligne que les habitants ne se sont sans doute pas préoccupés d'emporter dans leur fuite ces objets sans valeur), la présence de squelettes (même si bien entendu il peut d'agir de ceux de fugitifs étrangers à la maison), et l'état des enduits peints (Allison, Pompeian households, tableaux p. 193-194).

17 Sur la date de l'éruption : G. Stefani, M. Borgongino, « Intorno alla data dell'eruzione del 79 d.C. ", Rivista di Studi Pompeiani 12-13 (2001), p. 177-215, cependant récemment corrigé en ce qui concerne l'attestation épigraphique, par R. AвDy, “ The last coin in Pompeii : a re-evaluation of the coin hoard from the House of the golden bracelet », Numismatic Chronicle 173 (2013), p. 79-83. Les arguments fondés sur les données archéobotaniques subsistent toutefois.

18 On sait par une inscription du III ${ }^{\mathrm{e}}$ ou $\mathrm{IV}^{\mathrm{e}}$ siècle qu'une communauté de réfugiés d'Herculanum s'était installée à Naples : N. Monteix, “Pompéi et Herculanum, observatoires privilégiés de résiliences urbaines inachevées? „, in G. Djament-Tran, 
C'est ainsi que deux des plus célèbres visions préconçues sur les sites d'Herculanum et Pompéi doivent être abandonnées ${ }^{19}$. D’une part, la cité d'Herculanum n'a pas été “ figée » dans un instant de vie quotidienne et domestique ordinaires; d'autre part, les quelques objets et meubles découverts sur le site lors des fouilles, n'offrent pas une vision exhaustive du mobilier contenu dans les maisons. Ajoutons une troisième observation : les objets découverts ne se trouvaient pas nécessairement à l'endroit où ils étaient rangés habituellement, en temps normal ${ }^{20}$. Toutes ces réflexions font partie d'une réflexion globale sur les données disponibles et sur le potentiel de reconstruction, au moment de passer à l'étape de restitution informatique d'une habitation.

\section{Le passage à la restitution 3D d'un édifice}

Lors de la réalisation d'une restitution virtuelle d'un édifice d'Herculanum, trois niveaux de reconstruction sont mis en œuvre. Ils peuvent être classés selon le degré de relation aux sources sur lesquels ils s'appuient, et donc la fiabilité des hypothèses formulées :

1- Reproduire ce qui est connu. C'est le cas des sols du rez-de-chaussée qui présentent l'avantage d'être, bien souvent, la partie la mieux conservée de la maison. La restitution peut donc en être particulièrement fidèle et fiable.

2- Compléter les lacunes des éléments en partie présents mais altérés. Ce type d'intervention peut être envisagé pour compléter l'élévation d'un mur en partie effondré. Rentrent également dans cette catégorie les infographies de restitution des peintures murales altérées, ainsi que le montre l'article de Hélène Eristov et Marie-Laure Maraval présenté dans ce volume.

3- Combler les manques. De nombreux éléments de décor, d'architecture mais aussi de mobilier et d'instrumentum ont disparu. La cohérence et la lisibilité de l'ensemble ordonne qu'ils soient restitués, ou au moins évoqués. C'est le cas des charpentes, portes, tentures, etc.

La nécessité, pour l'archéologue, de proposer des évocations graphiques les plus rigoureuses mais aussi les plus attractives que possible, afin de mar-

M. Reghezza-Zitt (dir.), Résiliences urbaines. Les villes face à la catastrophe, Paris, 2012, p. 47-71 : Regio Primaria Herculanensium (CIL X, 1492).

19 C'est également ce que fait remarquer P.M. Allison en préambule de son ouvrage sur la culture matérielle des maisons pompéiennes : Allison, Pompeian households (supra n. 14), p.14-15.

20 Pour Pompéi, on pense notamment au cas de la maison du Ménandre dont les propriétaires avaient rangé à l'abri, dans un coffre placé dans une salle au sous-sol, toute leur argenterie (A. MAIURI, La Casa del Menandro e il suo tesoro d'argenteria, Rome, 1933, fig. 102). 
quer durablement l'imaginaire du public, a déjà été évoquée par ailleurs ${ }^{21}$. L'omniprésence, dans notre culture contemporaine, de représentations faussées - mais spectaculaires du point de vue graphique - de l'architecture antique, impose aux scientifiques d'offrir des restitutions aptes à rivaliser, du point de vue esthétique, avec les images diffusées par le cinéma ou les jeux vidéo, mais réalisées suivant une déontologie exemplaire. Cette impérieuse nécessité a d'ailleurs été actée par une partie de la communauté scientifique, donnant naissance en 2009 à la rédaction de la London Charter (http://www.londoncharter.org/) qui établit les normes de restitution informatique des édifices patrimoniaux fondées sur des critères scientifiques communs ${ }^{22}$.

Il faut ainsi éviter, autant que possible, de restituer les éléments manquants du décor ou du mobilier d'une maison par des emprunts à un autre édifice. Voilà pourquoi, dans la restitution virtuelle de la casa di Nettuno ed Anfitrite réalisée par l'équipe du programme ANR VESUVIA, en collaboration avec Archéovision, des “ éléments cliquables » permettront, à la consultation du modèle 3D, de justifier l'origine et les sources des restitutions de décor, ainsi qu'on le lira dans les contributions de Maud Mulliez et d'Hélène Eristov. En effet, s'agissant des résultats d'enquêtes archéologiques, historiques et philologiques, il est important de rendre accessibles, en lien direct avec ces images, les documents ayant permis de les réaliser : la mise à disposition des données, éventuellement ténues, sur lesquels se fondent nos hypothèses garantit la rigueur de la démarche.

\section{Des maisons sans habitants?}

L'espace domestique ne fait sens que peuplé de ses habitants, mais comment matérialiser leur présence au sein de reconstructions virtuelles? Une telle démarche implique une enquête préalable, afin d'estimer l'importance numérique de la cellule familiale.

Ainsi que le soulignait Lisa Nevett, les groupes sociaux vivant ensemble sous un même toit peuvent varier énormément dans leur composition ${ }^{23}$. Mais elle rappelle également qu'il n'existe pas ou peu de témoignages attestant l'existence d'unité domestique abritant une personne isolée dans l'Antiquité, alors que le phénomène est extrêmement courant dans les sociétés occidentales contemporaines. Dans les mondes grec et romain, une habitation peut accueillir une famille nucléaire

21 A. Dardenay et al., "Herculanum. Des archives aux restitutions architecturales et décoratives ", Chronique des activités archéologiques de l'École française de Rome (2016) (URL = http://cefr.revues.org/1588).

22 H. Denard, “ A New Introduction to the London Charter », in A. BentKowsKa-Kafel, D. Baker, H. Denard (éd.), Paradata and Transparency in Virtual Heritage, Ashgate, 2012, p. 57-71.

23 L.C. Nevetr, Domestic space in classical antiquity, Cambridge, 2010, p.16. 
réduite à un seul couple avec ses enfants, mais en fonction du niveau social de la maisonnée (housefull), elle peut aussi réunir des proches, amis, membres d'une même famille même éloignée, locataires, serviteurs ou esclaves.

Une des principales difficultés à laquelle nous sommes confrontés dans l'étude de la sphère domestique à Herculanum, comme à Pompéi, est l'évaluation du nombre d'habitants vivants dans la cité, et dans chaque habitation prise individuellement, au cours des années précédant l'éruption. Plusieurs modes de calcul ont été envisagés, offrant des fourchettes à la fois très larges et très différentes, d'une étude à l'autre ${ }^{24}$. Cette “ archéologie de l'invisible », appliquée à l'espace domestique a reçu récemment une interprétation très stimulante, dans l'ouvrage de S. R. Joshel et L. Hackworth Petersen portant sur « la vie matérielle » des esclaves romains ${ }^{25}$. Les auteurs déploient des outils analytiques innovants afin d'identifier dans l'architecture, le décor, les graffiti et autres subtiles traces matérielles, des indices des modalités d'occupation des espaces domestiques. C'est ainsi que la hauteur des portes dans une habitation, par exemple, peut être utilisée comme fil conducteur à une mise en évidence des stratégies de circulation, en fonction du statut (servile/libre) de ses occupants ${ }^{26}$, à l'intérieur de l'espace occupé par une familia.

S'il faut se résigner à l'absence de certitudes concernant le nombre d'occupants d'une maison, l'archéologie du bâti, combinée parfois à l'étude du matériel mis au jour, peut au moins permettre d'identifier le nombre d'unités d'habitation occupant une domus d'Herculanum. Le site présente la rare particularité de conserver une bonne partie des étages des édifices et offre donc un laboratoire d'étude idéal à ce type d'expérience planimétrique, les pièces à l'étage occupant un rôle important dans les stratégies d'investissement de l'espace habitable par la familia. Des travaux en ce sens ont d'ailleurs été initiés ces dernières années, et ils révèlent que les pièces aménagées au premier étage étaient bien souvent des espaces de confort et standing équivalents, voire supérieurs à ceux du rez-dechaussée ; ils pouvaient de plus participer à une « saisonnalité » de l'occupation de l'espace domestique, ainsi que le propose J. Andrews pour la casa d'Argo ${ }^{27}$.

24 Sur cette question, voir G. CAmodeca, «La popolazione degli ultimi decenni di Ercolano ", in M. Borriello et al. (dir.), Ercolano. Tre secoli di scoperte, 2008, p. 86-103. Il estime la population à un maximum de 4000 habitants, esclaves inclus, en se fondant sur les différentes inscriptions d'Herculanum, dont l'album CIL X, 1403 : ibidem, p. 67.

25 S.R. Joshel, L.H. Petersen, The material life of Roman slaves, New York, 2014 : "Invisilibity in Archaeology», p. 4-7.

26 Ibid., p. 37-59.

27 Anabases (26), 2017, p. 117-141. Sur l'occupation des étages voir aussi J.N. Andrews, The use and development of upper floors in houses at Herculaneum, Ph.D. thesis, 
Une autre question se pose, celle de la phase choisie pour la reconstruction 3D mise en œuvre. À Herculanum, plusieurs solutions de redécoupage ou de réagencement de l'espace intérieur des domus ont été opérées par les propriétaires successifs, afin de s'adapter à l'évolution de leur maisonnée et de leurs besoins ou à des contingences financières. S'il n'est pas souvent possible de donner une chronologie absolue de ces interventions, une chronologie relative des modifications successives est, en revanche, envisageable. Dès lors, au moment de la restitution 3D d'un édifice se pose inévitablement la question du " quand? ». Quel état de la maison doit-on restituer? On imagine que le dernier état avant destruction s'impose, surtout dans le cas d'un site comme Herculanum. Mais estil le plus pertinent? Et comment mobiliser et restituer les précieuses données issues de l'étude diachronique de l'édifice qui, seules peuvent expliquer et justifier la planimétrie finale d'une maison? L'idéal serait une interface permettant une vision diachronique des différentes phases d'un édifice. Ce genre d'approche serait envisageable via la mise en place d'un système SIG.

Finissons sur cette ultime question, qui intervient inévitablement dans toute entreprise de “ reconstruction virtuelle » d'un édifice : celle de l'état d'usure de l'édifice restitué. Faut-il le représenter neuf, paré de sa polychromie éclatante, ou bien arbitrer en faveur d'un " état d'usage », plus difficile à mettre en œuvre informatiquement, mais tellement plus réaliste? La seconde solution est bien entendu à privilégier, dans la mesure du possible, et avec toutes les incertitudes liées à l'origine de l'usure constatée sur les décors et les structures. Dans ce cas, les traces de réfection et de réparations anciennes, quand elles sont repérables, constituent une aide précieuse pour établir la chronologie relative de l'existant.

Les enjeux problématiques liés à la mise en œuvre de ces restitutions virtuelles, en trois dimensions, sont donc multiples. Le résultat final proposé par une équipe résulte de nombreux arbitrages, dans lesquels le savoir et la sensibilité de chacun des partenaires permet de créer une synergie particulière et un modèle unique. Mais pour les mêmes raisons, les arbitrages, surtout concernant le troisième niveau de reconstruction (“combler les manques », cf supra) sont souvent compliqués par des désaccords au sein de l'équipe scientifique d'un projet. Ces désaccords ne doivent pas être vécus comme une entrave à l'achèvement du modèle 3D, mais au contraire, à un enrichissement, et même à une mise en conformité avec les règles

soutenue à l'University of Reading, 2006, p. 96-103. Sur la question de la délimitation des unités d'habitation dans la domus, voir A. Dardenay, “ Decoration in Context. Decorative Programs in light of the Archaeology of Buildings from the insula V of Herculaneum ", in E.M. Moormann, S.T.A.M. Mols (dir.), Context and Meaning, Babesch Supplements, 2017, p. 283-290. 
déontologiques d'une reconstruction virtuelle, telles qu'énoncées dans la London Charter. Dans ce cas, une voie appropriée, et aisée à mettre en œuvre du point de vue informatique, consiste à proposer plusieurs solutions de restitutions pour les éléments équivoques.

Les différentes contributions proposées dans ce volume, ainsi que dans le précédent (Anabases $\left.\mathrm{n}^{\circ} 26,2017\right)$, permettent de revenir de manière plus approfondie sur toutes ces questions et de révéler des propositions innovantes pour résoudre nombre des problèmes posés à l'archéologue et à l'historien dans la « matérialisation virtuelle » du résultat de ses travaux.

\author{
Alexandra Dardenay \\ Université Toulouse - Jean Jaurès \\ Laboratoire TRACES, CNRS, UMR 5608 \\ 5, allées Antonio Machado \\ 31058 Toulouse \\ adardenay@yahoo.fr
}



Anabases 27 (2018), p. 53.

\section{Errata}

Errata relatifs à l'article de James Andrews, « Revisiting the upper floors of the casa di Argo at Herculaneum » paru dans le volume 26 d'Anabases, p. 117-141.

- Lire 'Casa d'Argo' en lieu et place de 'Casa di Argo' dans le titre et l'ensemble de l'article

- Page 128, note 29, les références correctes sont les suivantes :

Ruggiero, Storia degli scavi di Ercolano, p. 539 (21 Jan. 1828), 540, 542 (14 Feb. 1828). Bonucci's initial description of the shelving (21 Jan. 1828) includes the description of the storeroom in the general discussion of the large rectangular room, thereby not drawing a distinction between the two spaces. However, his subsequent account (14 Feb. 1828) indicates that the storeroom was at the end of the large room. Moreover, the plan provided on the same page is labelled as 'pianta della stanza e del ripostiglio descritti?

- Page 135, note 52, la référence complète doit être lue comme suit :

C. Bonucci, « Ercolano », Bollettino dell'Tstituto di Corrispondenza Archeologica (1829), p. 196. 
